\title{
Toe-brachial index is associated more strongly with albuminuria or glomerular filtration rate than ankle-brachial index in patients with type 2 diabetes
}

\author{
Michiaki Fukui $^{1}$, Muhei Tanaka ${ }^{1}$, Masahide Hamaguchi ${ }^{2}$, Takafumi Senmaru ${ }^{1}$, Kazumi Sakabe ${ }^{1}$, Mai Asano ${ }^{1}$, \\ Masahiro Yamazaki ${ }^{1}$, Goji Hasegawa ${ }^{1}$, Saeko Imai $^{3}$ and Naoto Nakamura ${ }^{1}$
}

The aim of this study was to investigate whether toe-brachial index (TBI) is more strongly associated with albuminuria or estimated glomerular filtration rate (eGFR) than ankle-brachial index (ABI), and thus is a more suitable tool for evaluating the association between peripheral artery disease (PAD) and diabetic nephropathy than $A B I$ in patients with type 2 diabetes. We evaluated the relationships between ABI or TBI and the degree of urinary albumin excretion or eGFR, as well as the major cardiovascular risk factors, in 390 patients with type 2 diabetes. Furthermore, we compared the area under the receiveroperator characteristic curve (AUC) of TBI or ABI for albuminuria or chronic kidney disease (CKD). Low-density lipoprotein cholesterol was negatively associated with ABI. Age and duration of diabetes were negatively associated with TBI, and diastolic blood pressure and high-density lipoprotein cholesterol were positively associated with TBI. Log (urinary albumin excretion) was associated more strongly with TBI $(r=-0.265, P<0.0001)$ than with $A B I(r=-0.132, P=0.0111)$, and eGFR was positively associated with TBI $(r=0.195, P=0.0002)$ but not with $A B I(r=0.023, P=0.6571)$. The AUCs of TBI for albuminuria $(P=0.0002)$ and CKD $(P=0.0322)$ were significantly greater than those of $A B I$. In conclusion, TBI is associated more strongly with albuminuria and eGFR than $A B I$ in patients with type 2 diabetes. Our study suggests that TBI may be a more suitable tool for evaluating the association between PAD and diabetic nephropathy than $A B I$ in patients with type 2 diabetes. Hypertension Research (2012) 35, 745-749; doi:10.1038/hr.2012.16; published online 16 February 2012

Keywords: cardiovascular disease; chronic kidney disease; peripheral artery disease

\section{INTRODUCTION}

Cardiovascular disease (CVD) is the leading cause of mortality and morbidity in patients with type 2 diabetes. ${ }^{1,2}$ Recently, chronic kidney disease (CKD) has been increasingly drawing attention as a high-risk factor for death from CVD, ${ }^{3,4}$ and diabetic nephropathy is a major cause of CKD. ${ }^{5}$ Moreover, an elevated albumin excretion rate, which is a useful marker of diabetic nephropathy, has been reported to be associated with increased risk of cardiovascular mortality. ${ }^{6}$ The anklebrachial index $(\mathrm{ABI})$ is a simple and useful method to assess peripheral artery disease (PAD). Furthermore, an ABI value $<0.9$ has been reported to be a risk factor for CVD events. ${ }^{7}$ However, application of this index to diabetic patients is considered questionable because of medial arterial calcification, which falsely elevates the ABI value. ${ }^{8,9}$ Therefore, toe-brachial index (TBI) is advocated for diabetic patients because medial arterial calcification is less frequent in the toe than in the ankle.

There has been no study that has simultaneously investigated the association between ABI or TBI and albuminuria or estimated glomerular filtration rate (eGFR). The aim of this study was to investigate whether TBI is more strongly associated with albuminuria or eGFR, and thus is a more suitable tool for evaluating the association between PAD and diabetic nephropathy than ABI in patients with type 2 diabetes who do not have advanced diabetic nephropathy.

\section{METHODS}

Patients

ABI and TBI were measured in 390 patients (241 men and 149 women) with type 2 diabetes recruited from the outpatient clinic at the Kyoto Prefectural University of Medicine. Type 2 diabetes was diagnosed according to the report of the Expert Committee on the Diagnosis and Classification of Diabetes Mellitus. ${ }^{10}$ Retinopathy was assessed with a state of mydriasis by ophthalmologists who were unaware of the data, and was graded as follows: no diabetic retinopathy, simple diabetic retinopathy and proliferative, including preproliferative, diabetic retinopathy. If the finding in the left and right fundi were discordant, the worse side was taken as a representative for the subject. Nephropathy was graded as follows: normoalbuminuria, urinary albumin excretion less than $30 \mathrm{mg}$ per gram of creatinine $\left(\mathrm{mgg}^{-1} \mathrm{Cr}\right)$; microalbuminuria, 30-300 $\mathrm{mgg}^{-1} \mathrm{Cr}$; or macroalbuminuria, more than $300 \mathrm{mg} \mathrm{g}^{-1} \mathrm{Cr}$. Sitting blood pressure was measured after a 5-min rest. CVD was defined as a

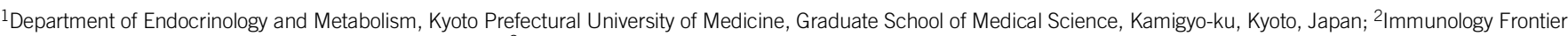
Research Center at Osaka University, Osaka, Japan and ${ }^{3}$ Department of Clinical Nutrition, Faculty of Comprehensive Rehabilitation, Osaka Prefecture University, Osaka, Japan Correspondence: Dr M Fukui, Department of Endocrinology and Metabolism, Kyoto Prefectural University of Medicine, Graduate School of Medical Science, 465 Kajii-cho, Kawaramachi-Hirokoji, Kamigyo-ku, Kyoto 602-8566, Japan.

E-mail: sayarinapm@hotmail.com

Received 25 October 2011; revised 10 January 2012; accepted 11 January 2012; published online 16 February 2012 
previous myocardial or cerebral infarction based on the clinical history or physical examination. Subjects were classified as nonsmokers, past smokers or current smokers according to a self-administered questionnaire. Patients with advanced renal dysfunction (serum $\mathrm{Cr}$ equal to or more than $2.0 \mathrm{mg} \mathrm{dl}^{-1}$ ) were excluded from this study.

\section{Experimental design}

We evaluated relationships of ABI or TBI to degree of urinary albumin excretion or eGFR, as well as to major cardiovascular risk factors, including age, blood pressure, serum lipid concentration and $\mathrm{Hb} \mathrm{Alc} \mathrm{(expressed} \mathrm{as} \mathrm{the}$ National GlycoHb Standardization Program values). We compared ABI or TBI according to sex, diabetic complications, smoking status and the presence of $\mathrm{CVD}$, insulin, pioglitazone, angiotensin-converting enzyme inhibitors (ACEI) and/or angiotensin II receptor blockers (ARB), calcium channel blockers, statins or anti-platelet agents. Moreover, we compared the degree of urinary albumin excretion or eGFR among groups determined by values of ABI (cutoff level was 0.9) and TBI (cutoff level was 0.6), as previously reported by Sheen et al. ${ }^{11}$ We performed multiple regression analysis to determine the contribution of the variables on TBI. Furthermore, we compared the area under the receiver-operator characteristic curve (AUC) of TBI or ABI for albuminuria, defined as urinary albumin excretion equal to or more than $30 \mathrm{mg} \mathrm{g}^{-1} \mathrm{Cr}$, or CKD, defined as eGFR less than $60 \mathrm{ml} \mathrm{min}^{-1}$ per $1.73 \mathrm{~m}^{2}$. This study was approved by the local Research Ethics Committee and was conducted in accordance with Declaration of Helsinki, and informed consent was obtained from all participants.

\section{Biochemical analysis}

Blood samples were obtained in the morning. Serum low-density lipoprotein cholesterol, high-density lipoprotein cholesterol and triglyceride concentrations were assessed using standard enzymatic methods. $\mathrm{Hb}$ Alc was assayed using high-performance liquid chromatography. Urinary albumin and $\mathrm{Cr}$ concentration were determined in an early-morning spot urine. Albumin excretion rate was measured with an immunoturbidimetric assay. A mean value for urinary albumin excretion was determined from three urine collections. GFR was estimated using the equation of Japanese Society of Nephrology: eGFR $=194 \times \mathrm{Cr}^{-1.094} \times \mathrm{age}^{-0.287}\left(\mathrm{ml} \mathrm{min}^{-1}\right.$ per $\left.1.73 \mathrm{~m}^{2}\right)$. For women, eGFR was multiplied by a correction factor of 0.739 .

\section{Measurement of ABI and TBI}

ABI and TBI were measured in 390 patients with type 2 diabetes using a Colin Waveform Analyzer (form PWV/ABI/TBI; Colin Medical Technology, Komaki, Japan), which simultaneously measures pulse volumes in the brachial and posterior tibial using an oscillometric method together with bilateral arm and ankle or toe blood pressure. ABI and TBI were measured after allowing the patient to rest in the supine position for at least $5 \mathrm{~min}$. Details of the method have been described elsewhere. ${ }^{12}$ The ABI or TBI was calculated bilaterally as the ratio of systolic pressure in the ankle or toe to systolic pressure in the arm, with the lower value considered as a representative for each subject. Intracoefficient of variation of ABI and TBI were 0.045 and 0.013 , respectively, and inter-coefficient of variation of $\mathrm{ABI}$ and TBI were 0.015 and 0.017 , respectively.

\section{Statistical analysis}

Means and frequencies of potential confounding variables were calculated. Unpaired Student's $t$-tests or analyses of variance followed by the Scheffe post hoc test were conducted to assess statistical significance of differences between groups. As urinary albumin excretion showed skewed distribution, logarithmic $(\log )$ transformation was carried out before performing correlation analysis. The relationships between ABI or TBI and log (urinary albumin excretion), eGFR, age, glycemic control or other variables were examined by Pearson's correlation analyses. In multiple regression analysis to assess the effects of variables on TBI, we included independent variables that were significantly correlated with TBI in the univariate analyses using SPSS version 11.0J (SPSS Inc. Chicago, IL, USA). Receiver-operator characteristic analyses were performed to calculate AUC of TBI or ABI for albuminuria or CKD using the ROCKIT (http://xray.bsd.uchicago.edu/krl/roc_soft.htm). All continuous variables are presented as the mean \pm s.d. A $P$-value $<0.05$ was considered statistically significant.

\section{RESULTS}

The characteristics of the 390 patients ( 241 men and 149 women) with type 2 diabetes enrolled in this study are shown in Table 1. Relationships between ABI or TBI and the characteristics of patients with type 2 diabetes are shown in Table 2. Low-density lipoprotein cholesterol was negatively associated with ABI. Age and duration of diabetes were negatively associated with TBI, and diastolic blood pressure and high-density lipoprotein cholesterol were positively associated with TBI. Log (urinary albumin excretion) was associated more strongly with TBI $(r=-0.265, P<0.0001)$ than with ABI $(r=-0.132, P=0.0111)$, and eGFR was positively associated with TBI $(r=0.195, P=0.0002)$ but not with ABI $(r=0.023, P=0.6571)$. ABI differed significantly according to diabetic nephropathy and differed between patients with and without CVD, statins or antiplatelet agents. TBI differed significantly according to diabetic nephropathy and smoking status, and differed between patients with and without CVD, statins or anti-platelet agents (Table 3).

TBI correlated significantly with ABI $(r=0.355, \quad P<0.0001$; Figure 1). Log (urinary albumin excretion) was significantly lower in a subgroup with normal ABI and normal TBI $(1.46 \pm 0.66)$ than that in a subgroup with normal ABI and low $(<0.6)$ TBI $(1.70 \pm 0.74$, $P=0.0079)$ and than that in a subgroup with low $(<0.9) \mathrm{ABI}$ and low TBI $(2.02 \pm 0.91, P=0.0117)$. Estimated GFR was significantly higher in a subgroup with normal ABI and normal TBI $(81.7 \pm 25.3)$ than that in a subgroup with normal ABI and low TBI $(74.0 \pm 22.1$, $P=0.0192)$ and than that in a subgroup with low ABI and low TBI $(65.3 \pm 28.0, P=0.0245)$.

\section{Table 1 Clinical characteristics of patients with diabetes}

\begin{tabular}{|c|c|}
\hline$n$ & 390 \\
\hline Sex (male/female) & $241 / 149$ \\
\hline Age (years) & $64.8 \pm 10.7$ \\
\hline Duration of diabetes (years) & $14.5 \pm 11.0$ \\
\hline Body mass index $\left(\mathrm{kg} \mathrm{m}^{-2}\right)$ & $23.5 \pm 4.0$ \\
\hline $\mathrm{Hb}$ Alc (\%) & $7.5 \pm 1.3$ \\
\hline Systolic blood pressure ( $\mathrm{mm} \mathrm{Hg}$ ) & $129 \pm 17$ \\
\hline Diastolic blood pressure (mm Hg) & $71 \pm 12$ \\
\hline LDL-cholesterol (mmol I-1) & $2.74 \pm 0.72$ \\
\hline Triglyceride $\left(\mathrm{mmoll}^{-1}\right)$ & $1.52 \pm 1.03$ \\
\hline HDL-cholesterol (mmol I-1) & $1.40 \pm 0.36$ \\
\hline Smoking (none/past/current) & $161 / 155 / 74$ \\
\hline Retinopathy (NDR/SDR/PDR) & $266 / 58 / 66$ \\
\hline Nephropathy (normo-/micro-/macroalbuminuria) & $212 / 127 / 51$ \\
\hline Neuropathy $(-/+)$ & $224 / 166$ \\
\hline Cardiovascular disease $(-/+)$ & $290 / 100$ \\
\hline Current treatment (Diet/oral hypoglycemic agents/insulin) & $51 / 209 / 130$ \\
\hline Urinary albumin excretion (mgg ${ }^{-1}$ creatinine) & $212 \pm 673$ \\
\hline Estimated glomerular filtration rate $\left(\mathrm{ml} \mathrm{min}^{-1}\right.$ per $\left.1.73 \mathrm{~m}^{2}\right)$ & $78.4 \pm 26.3$ \\
\hline Ankle-brachial index & $1.07 \pm 0.12$ \\
\hline Toe-brachial index & $0.68 \pm 0.17$ \\
\hline Pioglitazone $(-/+)$ & $343 / 47$ \\
\hline ACEI and /or ARB $(-/+)$ & $222 / 168$ \\
\hline Calcium channel blockers $(-/+)$ & $267 / 123$ \\
\hline Statins $(-/+)$ & $232 / 158$ \\
\hline Anti-platelet agents $(-/+)$ & $310 / 80$ \\
\hline
\end{tabular}

Abbreviations: ACEI, angiotensin-converting enzyme inhibitors; ARB, angiotensin II receptor blockers; HDL, high-density lipoprotein; LDL, low-density lipoprotein; NDR, no diabetic blockers; $\mathrm{HDL}$, high-density lipoprotein; $\mathrm{LDL}$, low-density lipoprotein; NDR, no diabetic
retinopathy; PDR, proliferative diabetic retinopathy; SDR, simple diabetic retinopathy. Data are mean \pm s.d. or number of subjects. 
In multiple regression analysis to examine the effects of variables on TBI, we included the following independent variables, which were significantly correlated with TBI in the univariate analyses: age, duration of diabetes, diastolic blood pressure, high-density lipoprotein cholesterol, smoking status, statins and anti-platelet agents. Multiple regression analysis demonstrated that age $(\beta=-0.335, P<0.0001)$, smoking status $(\beta=-0.173, P=0.0256)$ and diastolic blood pressure ( $\beta=0.201, P=0.0090)$ were independently associated with TBI. AUC (95\% confidence interval $(\mathrm{CI})$ ) of TBI and ABI for albuminuria were $0.652(0.596-0.708, P<0.001)$ and $0.530(0.470-0.590, P=0.316)$, respectively. AUC (95\% CI) of TBI for albuminuria was significantly greater than that of ABI $(P=0.0002$, Figure $2 a)$. AUC $(95 \% \mathrm{CI})$ of TBI and $\mathrm{ABI}$ for $\mathrm{CKD}$ were $0.623(0.553-0.694, P=0.001)$ and 0.523 (0.443-0.603, $P=0.545)$, respectively. AUC $(95 \% \mathrm{CI})$ of TBI for $\mathrm{CKD}$ was significantly greater than that of $\mathrm{ABI}(P=0.0322$, Figure $2 \mathrm{~b})$.

\section{DISCUSSION}

The present study demonstrated that log (urinary albumin excretion) was associated more strongly with TBI than with ABI, and eGFR was positively associated with TBI but not with ABI. The AUC (95\% CI) of

Table 2 Correlation between $\mathrm{ABI}$ or TBI and characteristics of patients with diabetes

\begin{tabular}{|c|c|c|c|c|}
\hline & \multicolumn{2}{|c|}{$A B I$} & \multicolumn{2}{|c|}{$T B I$} \\
\hline & r & $\mathrm{P}$ & r & $P$ \\
\hline Age & -0.076 & 0.1322 & -0.338 & $<0.0001$ \\
\hline Duration of diabetes & -0.021 & 0.6956 & -0.244 & $<0.0001$ \\
\hline Body mass index & -0.057 & 0.2636 & -0.002 & 0.9647 \\
\hline $\mathrm{Hb} A 1 \mathrm{c}$ & -0.007 & 0.8844 & -0.042 & 0.4088 \\
\hline Systolic blood pressure & -0.059 & 0.2461 & -0.088 & 0.0840 \\
\hline Diastolic blood pressure & 0.072 & 0.1560 & 0.253 & $<0.0001$ \\
\hline LDL-cholesterol & -0.159 & 0.0023 & 0.035 & 0.5062 \\
\hline triglycerides & -0.078 & 0.1234 & 0.071 & 0.1640 \\
\hline HDL-cholesterol & 0.094 & 0.0642 & 0.119 & 0.0188 \\
\hline Log (urinary albumin excretion) & -0.132 & 0.0111 & -0.265 & $<0.0001$ \\
\hline Estimated glomerular filtration rate & 0.023 & 0.6571 & 0.195 & 0.0002 \\
\hline
\end{tabular}

Abbreviations: $\mathrm{ABI}$, ankle-brachial index; $\mathrm{HDL}$, high-density lipoprotein; $\mathrm{LDL}$, low-density lipoprotein; TBI, toe-brachial index.
TBI for albuminuria or CKD was significantly greater than that of ABI. To our knowledge, this is the first report to investigate simultaneously the association between ABI or TBI and albuminuria or eGFR, and whether TBI is more strongly associated with albuminuria or eGFR, and thus is a more suitable tool for evaluating the association between PAD and diabetic nephropathy than $\mathrm{ABI}$ in patients with type 2 diabetes.

An ABI value $<0.9$ has been reported to be a risk factor for CVD events. ${ }^{7}$ However, application of this index to diabetic patients is considered questionable because of medial arterial calcification, which falsely elevates the ABI value. ${ }^{8,9}$ Previous reports have demonstrated that varying degrees of medial arterial calcification are common in diabetic patients. ${ }^{9}$ Therefore, TBI is advocated for diabetic patients because medial arterial calcification is less frequent in the toe than in the ankle. Brooks et al. ${ }^{13}$ demonstrated that the assessment of TBI is clearly the method of choice in the presence of overt calcification as defined by an $A B I$ value $>1.3$. By contrast, in the absence of such overt calcification, the differences between ABI and TBI for diabetic patients were in the same range as for control subjects, who were assumed to have no calcification. Instead of ABI, the measurement of

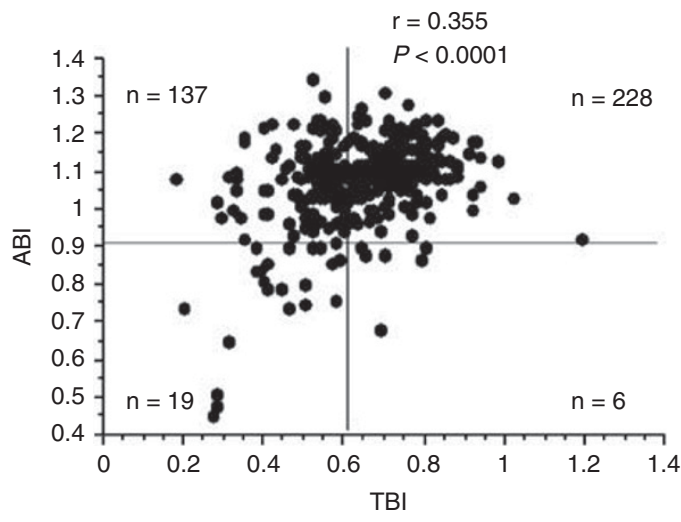

Figure 1 Correlation between toe-brachial index (TBI) and ankle-brachial index $(A B I)$, and classification of the study participants by $T B I$ and $A B I$. Abnormal $\mathrm{ABI}$ was defined as $<0.9$ and $\mathrm{TBI}<0.6$. TBI correlated significantly with $\mathrm{ABI}(r=0.355, P<0.0001)$.

Table 3 Comparisons of $\mathrm{ABI}$ or $\mathrm{TBI}$ in various groups

\begin{tabular}{|c|c|c|}
\hline Group & $A B I$ & $T B I$ \\
\hline Sex (male/female) & $1.08 \pm 0.13 / 1.07 \pm 0.10$ & $0.64 \pm 0.15 / 0.63 \pm 0.14$ \\
\hline Retinopathy (NDR/SDR/PDR) & $1.07 \pm 0.12 / 1.07 \pm 0.14 / 1.09 \pm 0.10$ & $0.64 \pm 0.15 / 0.66 \pm 0.16 / 0.60 \pm 0.15$ \\
\hline Nephropathy $(\mathrm{I} / \mathrm{II} / \mathrm{III})$ & $1.08 \pm 0.09^{a} / 1.07 \pm 0.11 / 1.04 \pm 0.16^{b}$ & $0.67 \pm 0.13^{c e} / 0.60 \pm 0.14^{d} / 0.56 \pm 0.14$ \\
\hline Neuropathy $(-/+)$ & $1.06 \pm 0.12 / 1.09 \pm 0.11$ & $0.63 \pm 0.16 / 0.64 \pm 0.14$ \\
\hline Smoking (none/past/current) & $1.08 \pm 0.10 / 1.05 \pm 0.10 / 1.05 \pm 0.17$ & $0.64 \pm 0.13 / 0.67 \pm 0.17 \mathrm{~g} / 0.58 \pm 0.14^{h}$ \\
\hline Cardiovascular disease $(-/+)$ & $1.09 \pm 0.10^{i} / 1.01 \pm 0.16^{j}$ & $0.65 \pm 0.13^{k} / 0.56 \pm 0.15^{1}$ \\
\hline Insulin $(-/+)$ & $1.07 \pm 0.13 / 1.07 \pm 0.12$ & $0.63 \pm 0.15 / 0.63 \pm 0.14$ \\
\hline Pioglitazone $(-/+)$ & $1.07 \pm 0.12 / 1.08 \pm 0.11$ & $0.64 \pm 0.15 / 0.63 \pm 0.13$ \\
\hline ACEI and/or ARB $(-/+)$ & $1.07 \pm 0.11 / 1.08 \pm 0.12$ & $0.64 \pm 0.16 / 0.63 \pm 0.13$ \\
\hline Calcium channel blockers $(-/+)$ & $1.08 \pm 0.10 / 1.06 \pm 0.15$ & $0.64 \pm 0.15 / 0.62 \pm 0.15$ \\
\hline Statins $(-/+)$ & $1.09 \pm 0.10^{m} / 1.05 \pm 0.14^{n}$ & $0.65 \pm 0.16 \% 0.61 \pm 0.13^{p}$ \\
\hline Anti-platelet agents $(-/+)$ & $1.08 \pm 0.10^{q} / 1.04 \pm 0.16^{r}$ & $0.65 \pm 0.15^{\mathrm{s}} / 0.58 \pm 0.14^{\mathrm{t}}$ \\
\hline
\end{tabular}

Abbreviations: $\mathrm{ABI}$, ankle-brachial index; ACEI, angiotensin-converting enzyme inhibitors; ARB, angiotensin II receptor blockers; NDR, no diabetic retinopathy; PDR, proliferative diabetic retinopathy; SDR, simple diabetic retinopathy; TBI, toe-brachial index. Nephropathy (I/II/III) indicates Nephropathy (normo-/micro-/macroalbuminuria). Data are mean \pm s.d.

${ }_{\text {ab }} P=0.0175,{ }^{\mathrm{cd}} P<0.0001$, ef $P<0.0001$, gh $P=0.0059$, ij $P<0.0001$, kl $P<0.0001,{ }^{\mathrm{mn}} P=0.0073$, op $P=0.0047$, ar $P=0.0017$, st $P=0.0002$. 

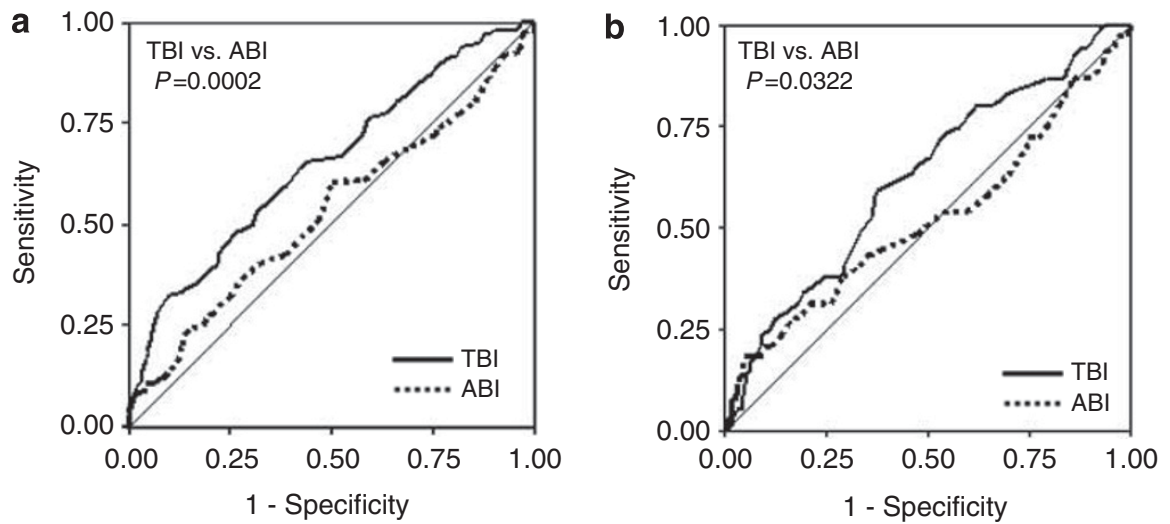

Figure 2 (a) Area under the receiver-operator characteristic curve (AUC) of toe-brachial index (TBI) and ankle-brachial index (ABI) for albuminuria defined as urinary albumin excretion equal to or more than $30 \mathrm{mgg}^{-1}$ creatinine. We compared AUC $(95 \%$ confidence interval $(\mathrm{CI}))$ between the two groups using the ROCKIT. TBI vs. ABI, $P=0.0002$. (b) $A U C$ of $T B I$ and $A B I$ for chronic kidney disease (CKD), defined as estimated glomerular filtration rate less than $60 \mathrm{ml} \mathrm{min}-1$ per $1.73 \mathrm{~m}^{2}$. We compared AUC $(95 \% \mathrm{Cl})$ between the two groups using the ROCKIT. TBI vs. ABI, $P=0.0322$.

TBI is recommended to diagnose PAD in diabetic patients, especially those with an ABI value $>1.3$. Usually, ABI was classified into three groups $(<0.9,0.9-1.3,>1.3)$, but not a continuous value. Continuous value may not be reliable in patients with type 2 diabetes. However, ABI was used as a continuous value in many previous reports. ${ }^{11,13-16}$ Therefore, we analyzed $A B I$ as a continuous value to certify that ABI was less strongly associated with albuminuria or eGFR, and thus is a less suitable tool in evaluating the association between PAD and diabetic nephropathy, than TBI in patients with type 2 diabetes. Furthermore, TBI was associated more strongly with albuminuria or eGFR than ABI after exclusion of patients with an ABI value $>1.3$ (data not shown).

Risk factors for the development of microalbuminuria are the same as those associated with vascular disease (endothelial dysfunction and inflammation). Microalbuminuria may be an early sign of intra-renal vascular dysfunction and a marker of vascular risk in the general population, as well as in high-risk individuals such as diabetes patients. ${ }^{17,18}$ The presence of PAD might imply atherosclerosis involving not only vasculatures of the heart, the brain and the lower limbs but also the vasculatures of the kidneys, which could cause intra-glomerular hypertension, glomerular damage and leakage of albumin. As the conditions associated with endothelial damage predispose individuals to microalbuminuria, PAD might be related to albuminuria. Traditional risk factors associated with PAD include smoking, hypertension, dyslipidemia, diabetes and CKD. Wu et al. ${ }^{19}$ demonstrated that the association between albuminuria and PAD (low $\mathrm{ABI}$ ) applied to nondiabetic but not to diabetic patients. TBI might be associated with albuminuria in diabetic patients because TBI was more strongly associated with albuminuria than $\mathrm{ABI}$ in our study.

Log (urinary albumin excretion) was associated more strongly with TBI than with ABI, and eGFR was positively associated with TBI but not with ABI. Sheen et al. ${ }^{11}$ reported that low eGFR was a major determinant of low TBI, as well as low ABI; however, eGFR was associated more strongly with TBI than with $\mathrm{ABI}$ in patients with type 2 diabetes. Moreover, Aso et al. ${ }^{16}$ reported that serum inflammatory marker, including C-reactive protein, showed significant negative correlation with TBI but not with ABI.

Interestingly, diastolic blood pressure was positively and strongly associated with TBI in univariate and multivariate analyses. The precise reason for this finding was uncertain. In general, diastolic blood pressure decreases as atherosclerosis progresses in the elderly.
We speculate that it is important to maintain diastolic blood pressure to preserve peripheral blood flow.

Blockade of the renin-angiotensin system by ACEI and/or ARB reduces urinary albumin excretion rate and thus confers renal and cardiovascular protective effects. ${ }^{20}$ Tseng et al. ${ }^{21}$ demonstrated that albuminuria negatively correlated with $\mathrm{ABI}$ and was associated with PAD in elderly diabetic patients not treated with ACEI and/or ARB; however, this association was not significant in patients treated with ACEI and/or ARB. In our study, TBI was associated more strongly with $\log$ (urinary albumin excretion) or eGFR than ABI even after exclusion of patients with type 2 diabetes treated with ACEI and/or ARB (data not shown).

The limitations of our study include that it was a cross-sectional study, that it assessed a relatively small number of patients and that the correlations between TBI and albuminuria or eGFR were weak, although they were statistically significant, to conclude that TBI is a more suitable tool in evaluating the association between PAD and diabetic nephropathy than $\mathrm{ABI}$ in patients with type 2 diabetes. In addition, we performed the ABI/TBI measurement only once in the daytime regardless of time of day, although ABI/TBI measurement varies depending on the time of day. However, to our knowledge, this is the first report to simultaneously investigate the association between $\mathrm{ABI}$ or TBI and albuminuria or eGFR, and whether TBI is more strongly associated with albuminuria or eGFR in patients with type 2 diabetes. We have analyzed a simple correlation between TBI or ABI and albuminuria or eGFR. We have not included log (urinary albumin excretion) and eGFR as independent variables in multiple regression analysis on TBI, because those are risk markers but not factors of PAD.

Clinically, the identification of PAD, which can be easily diagnosed in clinical practice at minimal cost, in diabetic patients is important because these patients are more prone to develop symptoms of claudication $^{22}$ and are at a higher risk of CVD. The clinical relevance relates to potential preventive and therapeutic approaches, whereas the diagnostic relevance concerns the diagnostic utility of TBI as a marker for PAD that can be measured easily and applied in clinical practice.

\section{Clinical implication}

TBI is advocated for diabetic patients because medial arterial calcification is less frequent in the toe than in the ankle. TBI is more strongly associated with albuminuria or eGFR than ABI, and thus is a more suitable tool for evaluating the association between PAD and diabetic 
nephropathy than $\mathrm{ABI}$, and $\mathrm{TBI}$ is recommended to be substituted for $\mathrm{ABI}$ for evaluating $\mathrm{PAD}$ in patients with type 2 diabetes.

\section{Conclusions}

TBI is associated more strongly with albuminuria or eGFR than ABI in patients with type 2 diabetes. Our study suggests that TBI may be a more suitable tool than ABI for evaluating the association between PAD and diabetic nephropathy in patients with type 2 diabetes.

\section{CONFLICT OF INTEREST}

The authors declare no conflict of interest.

\section{ACKNOWLEDGEMENTS}

We thank Dr Emi Ushigome, Yusuke Mineoka, Hisako Nakanishi, Hiroshi Okada, Shinobu Inada, Hiroya Iwase and Kanae Kobayashi for their assistance of researching data. All of them belong to Kyoto Prefectural University of Medicine.

1 Isomaa B, Almgren P, Tuomi T, Forsen B, Lahti K, Nissen M, Taskinen MR, Groop L. Cardiovascular morbidity and mortality associated with the metabolic syndrome. Diabetes Care 2001; 24: 683-689.

2 Multiple Risk Factor Intervention Trial Research Group. Multiple risk factor intervention trial: risk factor changes in mortality results. JAMA 1982; 248: 1465-1470.

3 Go AS, Chertow GM, Fan D, McCulloch CE, Hsu C. Chronic kidney disease and the risks of death, cardiovascular events, and hospitalization. N Engl J Med 2004; 351: 1296-1305.

4 Ninomiya T, Kiyohara Y, Kubo H, Tanizaki Y, Doi Y, Okubo K, Wakugawa Y, Hata J, Oishi Y, Shikata K, Yonemoto K, Hirakata H, lida M. Chronic kidney disease and cardiovascular disease in a general Japanese population: the Hisayama Study. Kidney Int 2005; 68: 228-236.

5 Hallan S, Dahl K, Oien CM, Grootendorst DC, Aasberg A, Holmen J, Dekker FW. Screening strategies for chronic kidney disease in the general population: follow-up of cross sectional health survey. BMJ 2006; 333: 1047-1050.

6 Dinneen SF, Gerstein HC. The association of microalbuminuria and mortality in noninsulin-dependent diabetes mellitus: a systematic overview of the literature. Arch Intern Med 1997; 157: 1413-1418.

7 Newman AB, Shemanski L, Manolio TA, Cushman M, Mittelmark M, Polak JF, Power NR, Siscovick D. Ankle-arm index as a predictor of cardiovascular disease and mortality in the Cardiovascular Health StudyGroup. Arterioscler Thromb Vasc Biol 1999; 19: 538-545.

8 Edmonds ME, Morrison N, Laws JW, Watkins PJ. Medial arterial calcification and diabetic neuropathy. BMJ 1982; 284: 928-930.

9 Young MJ, Adams JE, Anderson GF, Boulton AJ, Cavanagh PR. Medial arterial calcification in the feet of diabetic patients and matched non-diabetic control subjects. Diabetologia 1993; 36: 615-621.

10 The Expert Committee on the Diagnosis and Classification of Diabetes Mellitus. Report of the expert committee on the diagnosis and classification of diabetes mellitus. Diabetes Care 2002; 25: S5-S20.

11 Sheen YJ, Lin JL, Lee IT, Hsu YN, Li TC, Sheu WH. Low estimated glomerular filtration rate is a major determinant of low ankle-brachial index and toe-brachial index in type 2 diabetes. Angiology 2012; 63: 55-61.

12 Yamashina A, Tomiyama H, Takeda K, Tsuda H, Arai T, Hirose K, Koji Y, Hori S, Yamamoto $Y$. Validity, reproducibility, and clinical significance of noninvasive brachialankle pulse wave velocity measurement. Hypertens Res 2002; 25: 359-364.

13 Brooks B, Dean R, Patel S, Wu B, Molyneaux L, Yue DK. TBI or not TBI: that is the question. Is it better to measure toe pressure than ankle pressure in diabetic patients? Diabet Med 2001; 18: 528-532.

14 Uurtuya S, Taniguchi N, Kotani K, Yamada T, Kawano M, Khurelbaatar N, Itoh K, Lkhagvasuren T. Comparative study of the cardio-ankle vascular index and anklebrachial index between young Japanese and Mongolian subjects. Hypertens Res 2009; 32: 140-144.

15 Ali Z, Ellingtona AA, Mosley Jr TH, Kullo IJ. Association of serum osteoprotegerin with ankle-brachial index and urine albumin: creatinine ratio in African-Americans and nonHispanic whites. Atherosclerosis 2009; 206: 575-580.

16 Aso Y, Okamura K, Inoue T, Matsumoto R, Yoshida N, Wakabayashi S, Takebayashi K, Inukai T. Results of blood inflammatory markers are associated more strongly with toebrachial index than with ankle-brachial index in patients with type 2 diabetes. Diabetes Care 2004; 27: 1381-1386.

17 Gerstein HC, Mann JF, Yi Q, Zinman B, Dinneen SF, Hoogwerf B, Hallé JP, Young J, Rashkow A, Joyce C, Nawaz S, Yusuf S. Albuminuria and risk of cardiovascular events, death, and heart failure in diabetic and nondiabetic individuals. JAMA 2001; 286: 421-426.

18 Mimran A, Ribstein J, DuCailar G. Is microalbuminuria a marker of early intrarenal vascular dysfunction in essential hypertension? Hypertension 1994; 23: 1018-1021.

19 Wu CK, Yang CY, Tsai CT, Chiu FC, Huang YT, Lee JK, Cheng CL, Lin LY, Lin JW, Hwang JJ, Chiang FT. Association of low glomerular filtration rate and albuminuria with peripheral arterial disease: the Nationnal Health and Nutrition Examination Survey, 1999-2004. Atherosclerosis 2010; 209: 230-234.

20 American Diabetes Association Position Statement. Diabetic nephropathy. Diabetes Care 2004; 27: S79-S83.

21 Tseng CH, Tseng CP, Tai TY, Chong CK. Effect of angiotensin blockade on the association between albuminuria and peripheral arterial disease in elderly Taiwanese patients with type 2 diabetes mellitus. Circ J 2005; 69: 965-970.

22 Melzer D, Gardener E, Guralnik JM. Mobility disability in the middle-aged: crosssectional associations in the English longitudinal study of ageing. Age Ageing 2005; 34: 594-602. 